OPEN ACCESS

Edited by:

Quan Zou,

Tianjin University, China

Reviewed by:

Xiaoyong Pan,

Erasmus University Rotterdam,

Netherlands

Lin Lu,

Columbia University Irving Medical

Center, United States

${ }^{*}$ Correspondence:

Min Ye

gleye@163.com

tThese authors have contributed equally to this work

Specialty section:

This article was submitted to Bioinformatics and Computational

Biology,

a section of the journal

Frontiers in Genetics

Received: 03 August 2018

Accepted: 10 September 2018

Published: 08 October 2018

Citation:

Lu S, Zhao K, Wang X, Liu H, Ainiwaer $X, X u Y$ and Ye M (2018) Use of Laplacian Heat Diffusion Algorithm to Infer Novel Genes With Functions

Related to Uveitis.

Front. Genet. 9:425.

doi: $10.3389 /$ fgene.2018.00425

\section{Use of Laplacian Heat Diffusion Algorithm to Infer Novel Genes With Functions Related to Uveitis}

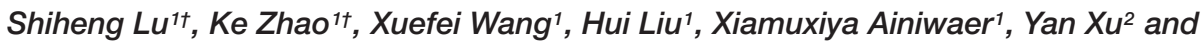 \\ Min Ye ${ }^{1 *}$ \\ ' Department of Ophthalmology, Shanghai Pudong Hospital, Fudan University Pudong Medical Center, Pudong, China, \\ ${ }^{2}$ School of Life Sciences, Shanghai University, Shanghai, China
}

Uveitis is the inflammation of the uvea and is a serious eye disease that can cause blindness for middle-aged and young people. However, the pathogenesis of this disease has not been fully uncovered and thus renders difficulties in designing effective treatments. Completely identifying the genes related to this disease can help improve and accelerate the comprehension of uveitis. In this study, a new computational method was developed to infer potential related genes based on validated ones. We employed a large protein-protein interaction network reported in STRING, in which Laplacian heat diffusion algorithm was applied using validated genes as seed nodes. Except for the validated ones, all genes in the network were filtered by three tests, namely, permutation, association, and function tests, which evaluated the genes based on their specialties and associations to uveitis. Results indicated that 59 inferred genes were accessed, several of which were confirmed to be highly related to uveitis by literature review. In addition, the inferred genes were compared with those reported in a previous study, indicating that our reported genes are necessary supplements.

Keywords: uveitis, Laplacian heat diffusion, protein-protein interaction, disease gene, network construction

\section{INTRODUCTION}

Uvea is a specific structure in the eyes and consists of the pigmented layer and the outer fibrous layer (Junqueira et al., 2013; Rekas et al., 2015). As one of the most common types of inflammation in uvea, uveitis is the third leading cause of blindness in all developed countries, generally affecting people aging 20-50 years (Junqueira et al., 2013). According to the statistics from the National Eye Institute in the United States, uveitis can be subtyped as anterior uveitis, intermediate uveitis, posterior uveitis, and panuveitis uveitis based on different pathogenic progressions and sites (Lim et al., 2016). Regardless of its subtype, the major pathogenesis of this disease is the over-activation of inflammatory cells in situ (Pan et al., 2014; Rosenbaum, 2015). Uvea contains most of the eye's blood vessels, from which immune cells can enter the eye. Therefore, uvea gets inflamed easier than other eye tissue regions, revealing the histological causes of uveitis' high morbidity rate.

The following five major clinical symptoms occur during the initiation and progression of uveitis: painful eye(s), bloodshot eye(s), sensitivity to light, and cloudy vision and floaters (Urban et al., 2014). In the early stage of such disease, the patient's eye(s) can only manifest as redness and conjunctivitis with no visual defeats. With the quick progression of such disease, blindness and the five common complications, including glaucoma, cataracts, optic nerve damage, retinal detachment, and permanent vision loss, are usually identified in the patient population 
(Sen et al., 2015). For the detailed pathogenic cause of such disease, the specific cause of uveitis in most clinical cases cannot be clearly identified due to its complicated candidate pathogenesis. In general, the top five causes of uveitis at the histological and pathogenic level can be clustered into five subgroups as follows: (1) eye injury, surgery; (2) autoimmune disorder; (3) inflammatory disorder; (4) eye tissue specific infection; and (5) cancer (Kalinina Ayuso et al., 2014). All such causes can be summarized as exogenous environmental effects and endogenous genetic contributions. According to recent publications, genetic and infectious contributions have received increasing attention and are widely regarded as the top two major pathogenic factors. Infectious progressions and their related immune responses of various infections, including brucellosis (Akyol et al., 2015), herpesviruses (Bojanova et al., 2013), and leptospirosis (Loureiro et al., 2013), contribute to the progression of uveitis, thus reflecting the unique pathogenic contribution of exogenous factors for uveitis.

Apart from exogenous factors such as infections, genetic contributions are also a major pathogenesis of uveitis. Early in 2014, a functional gene named FOXO1 has been confirmed to participate in the pathogenesis of acute anterior uveitis, thus reflecting the specific endogenous role of such gene for uveitis ( $\mathrm{Yu}$ et al., 2014). In 2015, another study on acute anterior uveitis confirmed that a specific immune associated gene, which is named C5 and encodes complement C5, contributes to its immune associated pathogenesis, thereby reflecting the complicated pathogenesis of such disease (Xu et al., 2015). Other complement associated genes and interleukin related genes have also been identified in different subtypes of uveitis, confirming the pathogenic genetic contribution of such disease (Yang et al., 2012). In 2015, a specific clinical trial (Ildefonso et al., 2015) on the gene therapy on uveitis revealed that the modification and recruitment of specific protein domains encoded by functional genes can reduce the ocular inflammatory response and relieve the symptoms. This finding indicated that genetic contributions may at least be one of the major pathogenic factors of uveitis.

Identifying the core pathogenic genetic factors and revealing the detailed pathogenic mechanisms based on experimental routines are difficult because of the organizational specificity (eye), relatively low incidence, and complicated pathogenesis of uveitis. In recent years, more and more computational methods (Tang et al., 2017; Zeng et al., 2017, 2018; Chen et al., 2018b; Pan et al., 2018; Wang et al., 2018) have been designed to investigate different diseases, thereby giving help to uncover pathogenic mechanisms of diseases. For uveitis, in 2017, uveitisrelated genes were identified based on a computational method (Lu et al., 2017), which adopted the classic network algorithm named random walk with restart (RWR) algorithm (Kohler et al., 2008; $\mathrm{Li}$ and $\mathrm{Li}, 2012$ ) to search novel genes in a protein-protein interaction (PPI) network. In the present study, we employed another network algorithm named Laplacian heat diffusion (LHD) to build a new computational method for inferring novel uveitis-related genes. LHD algorithm has different principles compared with RWR algorithm and thus may help us extract novel genes that cannot be identified by the method in Lu et al. (2017). In addition, the proposed method also adopted several tests to screen out the most related genes. Finally, 59 genes were accessed by our method, and only two of them were also reported in previous studies as validated pathogens (Lu et al., 2017). We conducted an extensive analysis on several of these genes to show the reliability of our method. The new findings reported in the present study may aid in revealing the detailed pathogenic mechanisms of uveitis.

\section{MATERIALS AND METHODS}

\section{Uveitis-Related Genes}

We extracted uveitis related genes from literature indexed by PubMed ${ }^{1}$. In the search bar, we set "uveitis" and "genes" as the keywords, thus obtaining 744 published articles. Among these articles, 98 were review articles, in which several solid uveitis related genes were reported. A total of 121 genes were selected by manual reviewing. These genes are important for uveitis or specific uveitis symptoms and thus were called uveitis-related genes in this study. Proteins encoded by the 121 uveitis-related genes were obtained and further mapped onto their Ensembl IDs because we adopted the PPI network to infer novel uveitis related genes based on these genes. Finally, 113 Ensembl IDs were obtained. The 121 uveitis genes and the Ensembl IDs of their proteins are provided in Supplementary Table $\mathbf{S} 1$.

\section{Construction of PPI Network}

PPI information is a useful material to study different proteinor gene-related problems (Hu et al., 2011a,b; Gao P. et al., 2012; Gao Y.F. et al., 2012; Chen et al., 2016a, 2018c; Huang et al., 2016; Zhang et al., 2016; Cai et al., 2017; Lu et al., 2017; Li et al., 2018a). Most studies using this information reported that two proteins that interact with each other always share similar functions. The proteins encoded by uveitis-related genes may have some common functions, which may also be shared by their interactive proteins. This procedure can further continue. If we start from the proteins encoded by uveitisrelated genes and diffuse their status to their neighbors and neighbors' neighbors, then some novel proteins that are strongly associated with proteins encoded by uveitis-related genes can be extracted. Their genes may be novel uveitis-related genes. We need a PPI network to complete these procedures. Here, we used the PPI network reported in STRING ${ }^{2}$ (version 10.0). Compared with PPI networks reported in other databases, such as DIP (Database of Interaction Proteins) database (Xenarios et al., 2000) and BioGRID (Stark et al., 2006), which are constructed using experimentally determined PPIs, the PPI network used in this study further contains functional associations between proteins. The PPIs in STRING were collected from the following sources: (1) genomic context predictions; (2) high-throughput lab experiments; (3) (conserved) co-expression; (4) automated text mining; and (5) previous knowledge in databases. Thus, they can widely measure the associations between proteins, providing more opportunities to infer novel uveitis-related genes. The file

\footnotetext{
${ }^{1}$ http://www.ncbi.nlm.nih.gov/pubmed/

${ }^{2}$ https://string-db.org
} 
"9606.protein.links.v10.txt.gz" was retrieved from STRING to construct this PPI network. In this file, large numbers of human PPIs were included. Each PPI was assigned a score to indicate its strength. The constructed PPI network termed proteins, represented by Ensembl IDs, as nodes. Two nodes were adjacent if and only if their corresponding proteins can interact with each other. Furthermore, each edge in the PPI network was assigned a weight, which was defined as the score of its corresponding PPI. For easy description, the constructed PPI network was called as $N$ in the following text.

\section{Method for Inferring Novel Uveitis-Related Genes}

Inferring novel genes related to different diseases in network level has become quite popular (Barabasi et al., 2011). Several classic network algorithms, such as shortest path algorithm (Gormen et al., 1990; Gui et al., 2015; Chen et al., 2016a; Zhang et al., 2016; Cai et al., 2017, Chen et al., 2018a), and RWR algorithm (Chen et al., 2017a, 2018c; Li et al., 2017, 2018a; Yuan and Lu, 2017; Zhang et al., 2017), have been applied to develop different computational methods in this regard. A recent publication (Lu et al., 2017) proposed a RWR-based computational method to identify novel uveitis-related genes and reported several ones. Another classic network algorithm, LHD algorithm (Leiserson et al., 2015), was employed to construct a novel computational method to infer novel uveitis-related genes that were not reported in Lu et al. (2017).

\section{LHD Algorithm}

As a classic network diffusion algorithm, heat diffusion algorithm always starts from some nodes, called seed nodes, and transmits predefined heats on these nodes to other nodes in the network. A heat assigned to a node represents the strength of the associations between the node and seed nodes. Here, we adopted one kind of heat diffusion algorithm named LHD (Leiserson et al., 2015) to infer novel uveitis-related genes in PPI network $N$. The brief description of LHD algorithm was as below.

Given a PPI network $N$, let $A$ be its adjacent matrix and $D$ is a diagonal matrix storing the degree of each node. The graph Laplacian $L$ was defined as $D$-A. According to the 113 Ensembl IDs, which were assigned to uveitis-related genes and used as seed nodes in LHD algorithm, an original heat distribution vector $H_{0}$ can be constructed in a way that the components corresponding to seed nodes were set to $1 / 113$ and others were set as zero. The heat distribution vector at time $t$ can be accessed by

$$
H_{t}=H_{0} \bullet \exp (-L t)
$$

where $\exp ($ ) is the matrix exponential. By setting a series of increasing values of $t$, we can obtain a series of heat distribution vectors. When two consecutive distribution vectors are quite similar, one vector is assigned as the output of LHD algorithm. In the output vector, each node, including seed nodes, received a heat value. We only extracted the heat values of non-seed nodes, which would be further used for selecting important genes.

\section{Permutation Test}

Each node received a heat value based on the LHD algorithm. However, this value may be affected by the structure of the PPI network $N$, i.e., some nodes may have high probabilities to receive high heat values regardless of which nodes are selected as seed nodes. Therefore, these nodes should not be selected as candidate genes of uveitis. To control this type of nodes, we performed a permutation test. We constructed 500 Ensembl ID sets, each comprising 113 Ensembl IDs that were randomly selected from the nodes in the PPI network $N$. For each set, the nodes were taken as seed nodes, which were inputted onto the LHD algorithm. After the 500 sets were tested, each node received several heat values. By comparing these values with the heat value obtained by using 113 Ensembl IDs of uveitis-related genes, we can compute a measurement, called zscore, to evaluate the reliability of the actual heat value. Zscore can defined as below:

$$
\text { zscore }(g)=\frac{h(g)-\mu(g)}{\delta(g)}
$$

where $h(g)$ is the heat value of gene $g$ obtained by 113 Ensembl IDs of uveitis-related genes, and $\mu(g)$ and $\delta(g)$ are the mean and standard deviation, respectively, of the heat values obtained by 500 randomly produced sets. According to statistical theory, 1.96 is the threshold for selecting genes that significantly correlate with uveitis-related genes. Thus, we extracted Ensembl IDs with zscores no less than 1.96. These IDs would be further analyzed by the following tests.

\section{Interaction Test}

Large numbers of genes were discarded through the permutation test. For the remaining genes, some were strongly associated with uveitis-related genes, indicating their high relationships to uveitis. By contrast, others had few, even no associations with uveitis-related genes, implying that they were not related to uveitis and should be discarded. To indicate the association between the candidate genes passing the permutation test and the uveitis-related genes, we employed the PPI information mentioned in Section "Construction of PPI Network". The score was also used to quantify the strength of the PPI. The score of the PPI of proteins $p_{1}$ and $p_{2}$ was denoted by $S\left(p_{1}, p_{2}\right)$. For one candidate gene $g$, we assigned a measurement called maximum interaction score (MIS), which was defined as:

$$
\operatorname{MIS}(g)=\max \left\{S\left(g, g^{\prime}\right): g^{\prime} \text { is a uveitis related gene }\right\}
$$

A high MIS indicates that the gene was highly related to at least one uveitis-related gene. Accordingly, this specific gene may also share the functions shared by this uveitis-related gene and thus has a high probability to become a novel uveitis-related gene. According to the setting in STRING, 900 was the threshold of highest confidence and was also set as the threshold of MIS, i.e., candidate genes passing the permutation test were retained if their MISs were no less than 900.

\section{Function Test}

The last test measured the linkage between candidate genes and uveitis-related genes based on gene ontology (GO) terms and 
biological pathways. Each gene has special relationships with some GO terms or pathways. If one candidate gene exhibited GO terms or pathways that are similar to one uveitis related gene, then it may be highly related to this uveitis-related gene and thus shows a high probability of being a novel uveitis gene. To complete this test, we employed a scheme to indicate the relationship between a gene and a GO term (pathway). Here, we adopted the GO and KEGG enrichment theory (Chen et al., 2016b, 2017a,b, 2018c; Liu et al., 2016; Lu et al., 2017; Li et al., 2018a,b), which can transform the relationship between one gene and one GO term (KEGG pathway) into a number. A vector, denoted as $E S(g)$, can be obtained by collecting the numbers of one gene $g$ between all GO terms and KEGG pathways. We further used the direction cosine of two vectors $E S(g)$ and $E S\left(g^{\prime}\right)$ to measure the linkage between $g$ and $g^{\prime}$ in terms of GO terms and KEGG pathways. The direction was defined as:

$$
C\left(g, g^{\prime}\right)=\frac{E S(g) \bullet E S\left(g^{\prime}\right)}{\|E S(g)\| \bullet\left\|E S\left(g^{\prime}\right)\right\|}
$$

where $E S(g) \bullet E S\left(g^{\prime}\right)$ is the dot product of two vectors and $\|E S(g)\|$ is the modulus of the vector $E S(g)$. A high outcome of equation 4 suggested strong associations between two genes. We assigned the last measurement named maximum function score (MFS), which is similar to MIS, to the candidate gene $g$. MFS can be computed by:

$$
\operatorname{MFS}(g)=\max \left\{C\left(g, g^{\prime}\right): g^{\prime} \text { is a uveitis related gene }\right\}
$$

Then, 0.97 was set as the threshold of MFS to extract final candidate genes. For convenience, the final obtained genes were called inferred genes.

\section{RESULTS}

In this study, we set up a computation method to infer novel uveitis-related genes based on validated ones retrieved from published literature. All procedures are shown in Figure 1. This section gave the detailed results of this method.

The Ensembl IDs for uveitis-related genes were picked up as seed nodes for LHD algorithm. Except for the uveitis-related ones, all genes were assigned heat values that are available in Supplementary Table S2. However, these values may be affected by the structure of the PPI network N. Genes receiving high heat values were not always highly related to uveitis. Accordingly, a permutation test was performed. Measurement zscores were computed and assigned to each candidate gene and are also available in Supplementary Table S2. According to Section "Permutation Test", we selected genes with zscores no less than 1.96 , accessing 1,287 candidate genes.

For the 1,287 candidate genes, we further filtered them by interaction test. The measurement MIS was calculated and assigned to each of these genes and is listed in Supplementary Table S2. We set 900 as its threshold, resulting in 391 candidate genes. In the function test, each of the 391 candidate genes was evaluated by MFS (see Supplementary Table S2). We set 0.97 as the threshold and finally obtained 59 genes (see first 59 genes

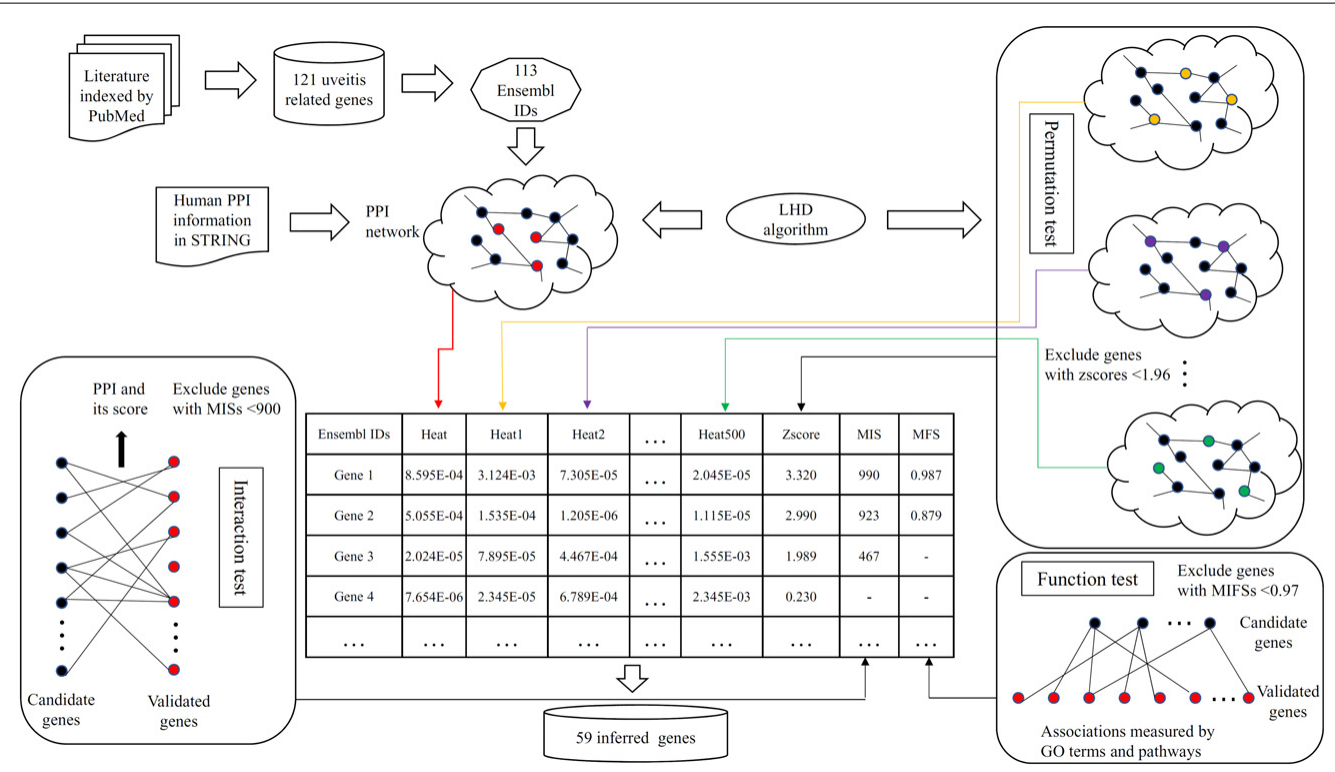

FIGURE 1 | Flow chart showing the detailed procedures of the computational method for inferring novel uveitis-related genes. Ensembl IDs of uveitis-related genes were used as the seed nodes of Laplacian heat diffusion (LHD) algorithm, resulting in a heat value for each gene in the PPI network. In the permutation test, the LHD algorithm was executed 500 times with different seed nodes, yielding 500 heat values for each gene. Then, a zscore (cf. equation 2) of each gene was calculated and those with zscores less than 1.96 were discarded. The interaction test assessed each candidate gene by checking its associations to validated genes and excluded candidate genes with MISs (cf. equation 3) less than 900 . Finally, the remaining genes were evaluated in the function test, which measured candidate genes by investigating their linkages based on gene ontology (GO) terms and biological pathways. Candidate genes with MFSs (cf. equation 5) less than 0.97 were discarded. Fifty-nine inferred genes were obtained. 


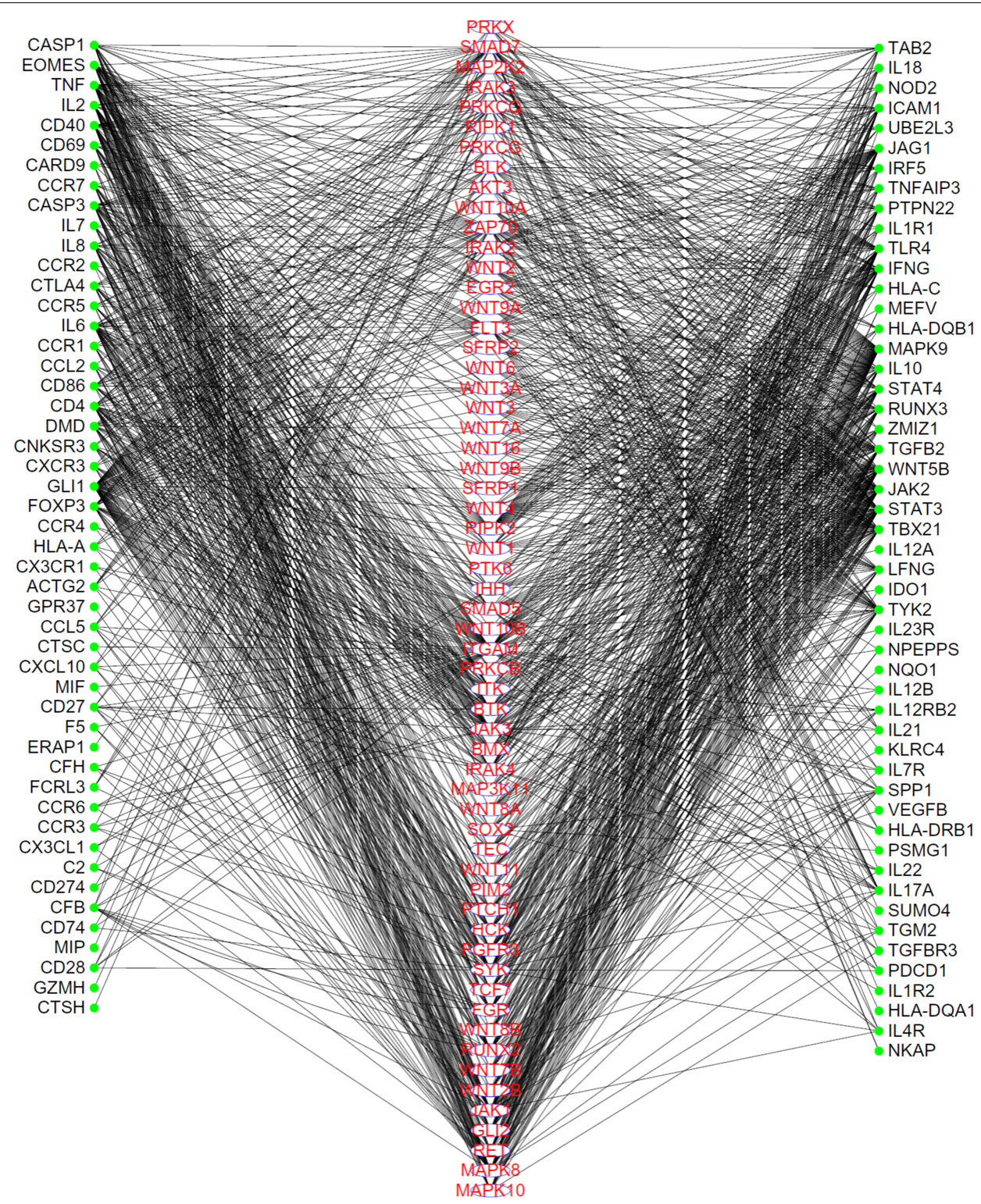

FIGURE 2 | The linkages between inferred uveitis-related genes and validated ones, which were extracted from the PPI network in STRING. Genes in the middle column are inferred genes, while genes in the left or right columns are validated genes. Each inferred gene has strong associations to validated genes.

in Supplementary Table S2). These genes were regarded to be highly related to uveitis and thus called inferred genes.

To show the high probabilities of inferred genes being novel uveitis-related genes, we extracted the linkages between 59 inferred genes and validated uveitis-related genes from the PPI network, as shown in Figure 2. It can be seen that each of these genes have strong associations with validated genes, proving that they can be novel uveitis-related genes.

\section{DISCUSSION}

In this study, we set up a new computational method for inferring novel uveitis-related genes. The method finally produced 59 inferred genes. This section first presents a comparison of the resulting genes with those reported in a previous study (Lu et al., 2017) and then gives an extensive analysis on several inferred genes.

\section{Comparison With Genes Reported in a Previous Study}

A previous study ( $\mathrm{Lu}$ et al., 2017) reported 56 novel genes that were related to uveitis and were accessed by using RWR algorithm and some screening tests. In the present study, 59 inferred uveitis-related genes were finally obtained. The Venn diagram on two gene sets, consisting of novel genes in Lu et al. (2017) and inferred genes in our study, is shown in Figure 3. 


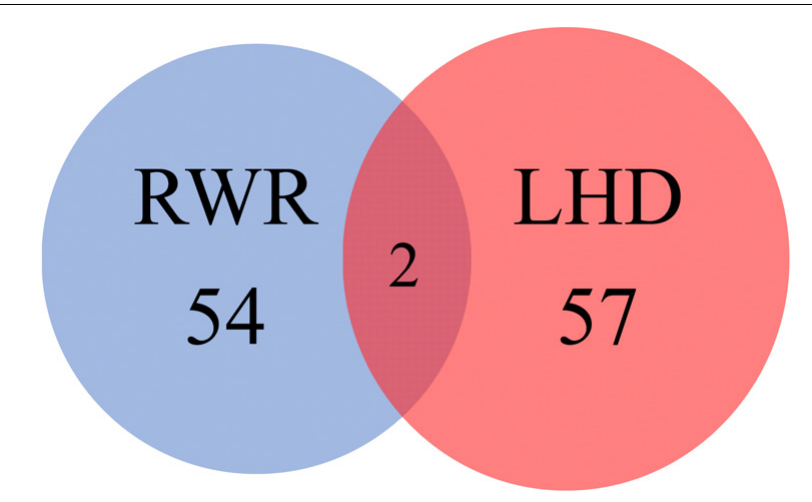

FIGURE 3 | Venn diagram illustrating two gene sets, consisting of novel genes in a previous study and inferred genes reported in this study. The blue circle represents the gene set consisting of novel genes in a previous study, which adopted RWR algorithm to search novel genes; while the red circle represents the gene set containing the inferred genes reported in this study, which were identified by LHD algorithm.

We can see that only two genes, JAK1 (ENSP00000343204) and MAPK8 (ENSP00000353483), were identified by both methods. The Jaccard coefficient of these two sets was $1.77 \%$, implying that the novel genes yielded by two methods were quite different. In addition, our inferred genes can be important supplements for the previous study if we can prove them to be highly related to uveitis. This result would be elaborated in the following subsection.

\section{Analysis of Inferred Genes}

Our computational method identified 59 inferred genes and regarded them to be highly related to uveitis. To confirm this, we did the GO and KEGG enrichment on them using R program clusterProfiler for detailed functional annotation. The results are provided in Supplementary Tables S3, S4. It can be observed that the inferred genes are functionally enriched in some $\mathrm{GO}$ terms, such as frizzled binding (GO:0005109), G-protein coupled receptor binding (GO:0001664), non-membrane spanning protein tyrosine kinase activity (GO:0004715), and protein tyrosine kinase activity (GO:0004713). The enriched KEGG pathways included signaling pathways regulating pluripotency of stem cells (hsa04550), Wnt signaling pathway (hsa04310), and melanogenesis (hsa04916).

According to recent publications, all inferred genes can be proved to be related to such disease or related pathogenic processes (Supplementary Table S5). Here, we selected important ones for detailed analyses, their detailed information is listed in Table 1. According to the enrichment analysis, we clustered these genes into three functional groups, as shown in Figure 4.

\section{Genes With Protein Tyrosine Kinase Activity (GO:0004713)}

The first inferred gene is a functional immune cell specifically expressed gene JAK3 (ENSP00000391676) (Lee et al., 2012) and is pathologically related to autosomal severe combined immunodeficiency disease (Notarangelo et al., 2001; Bogaert et al., 2016). For its specific contribution on the pathogenesis of uveitis, a specific study (Liao et al., 2018) on ankylosing spondylitis confirmed that the expression levels of JAK1 and JAK3 are positively correlated with various autoimmune diseases, including enthesitis, ankylosing spondylitis and uveitis, thereby validating their potential pathogenic contributions.

Apart from JAK3, another gene named as JAK1 (ENSP00000343204) was also inferred to be a potential pathogenic uveitis-related gene by our method. Based on the publications mentioned above, such gene was also confirmed to participate in uveitis-related pathogenesis.

Apart from that, the next inferred gene was BTK (ENSP00000308176). BTK contributes to the regulation of B cell maturation and proliferation under physical or pathological

TABLE 1 | Sixteen important inferred uveitis-related genes.

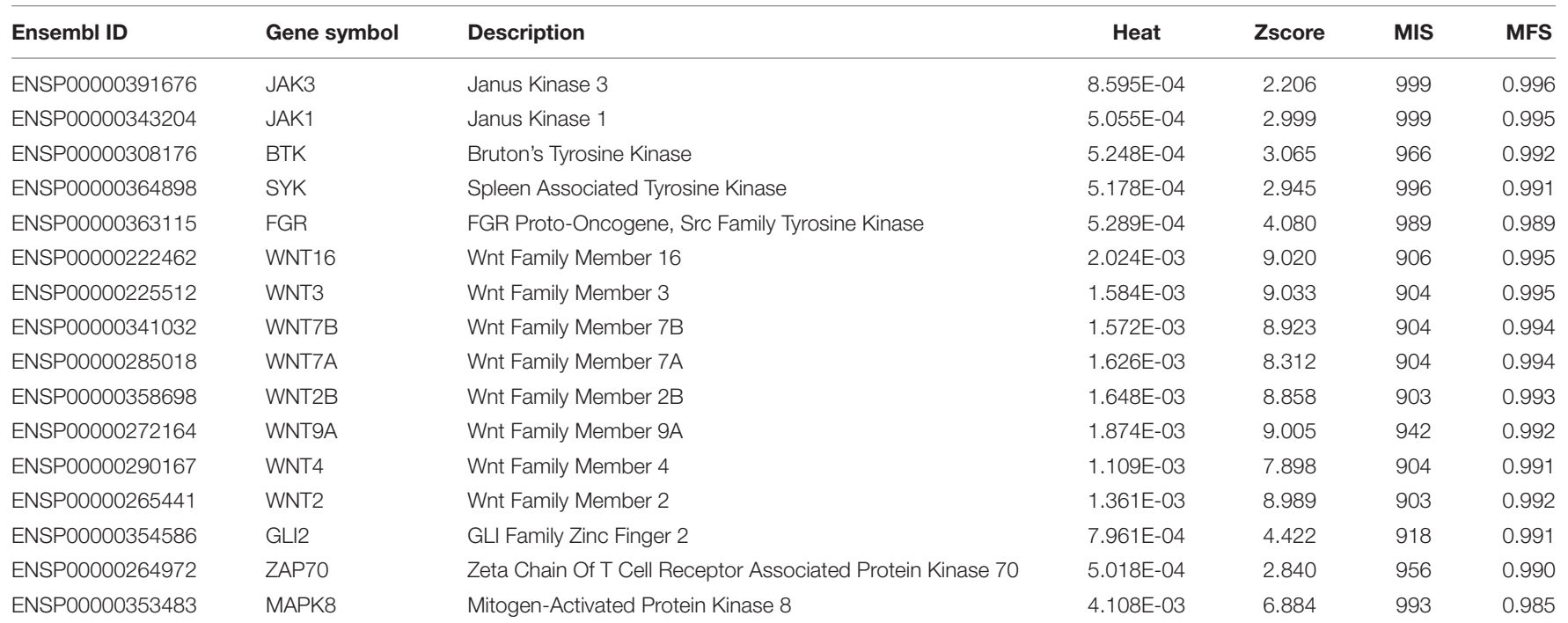




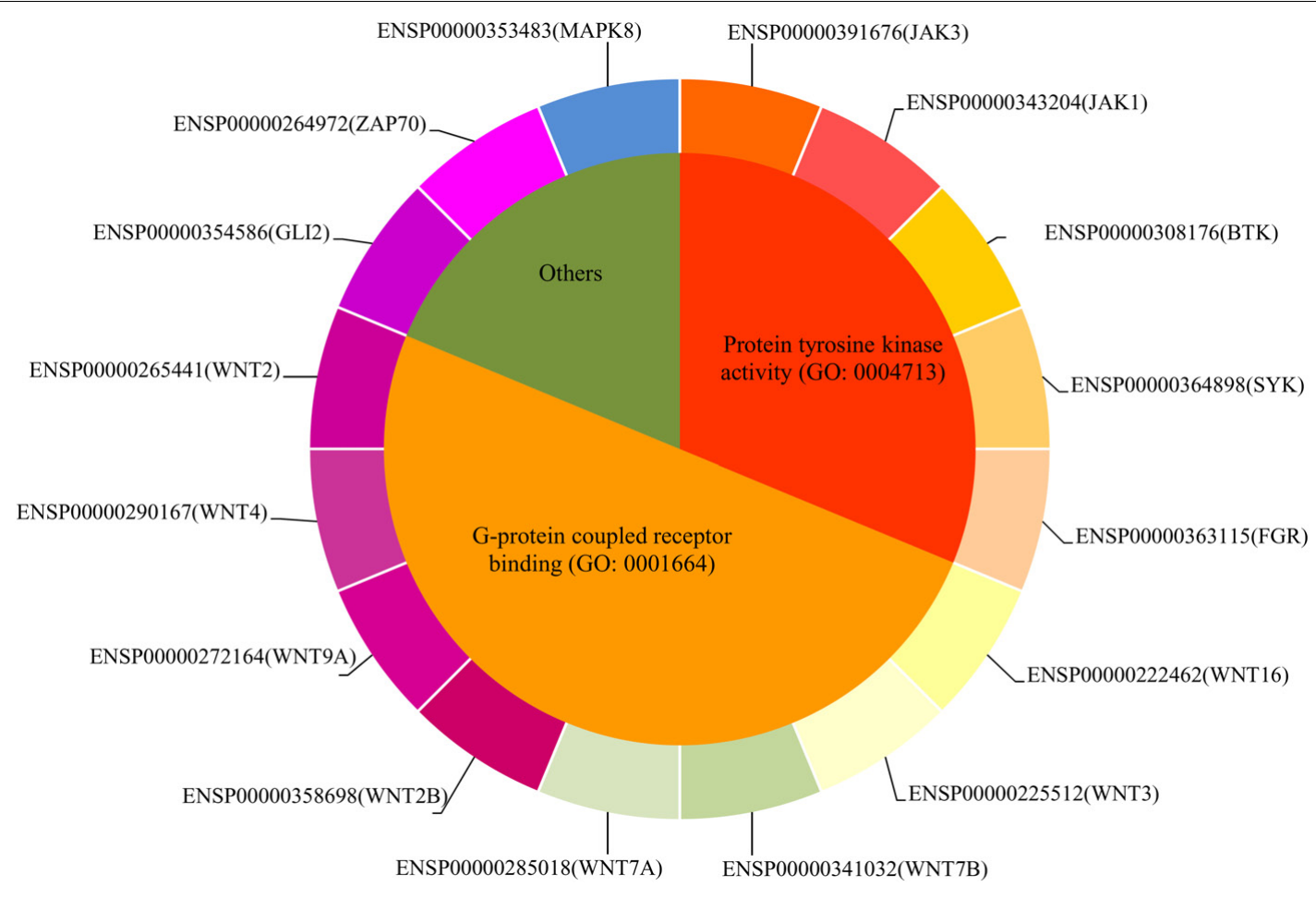

FIGURE 4 | The distribution of sixteen important inferred genes.

conditions (Wu et al., 2014; Nagel et al., 2015). According to recent publications, BTK is pathologically connected to uveitis by interfering immune cell differentiation (Vargas et al., 2013) and autoimmune responses (Corneth et al., 2016).

The next gene was SYK (ENSP00000364898), which contributes to the regulation of cellular responses, including proliferation, differentiation, and phagocytosis in $\mathrm{B}, \mathrm{T}$ and myeloid cells (Luger et al., 2013; Hauck et al., 2015; Wang et al., 2015). For its specific contribution on the pathogenesis of uveitis, SYK/CARD9 signaling axis participates in the pathogenesis of autoimmune eye diseases, including ocular inflammatory disorders, uveitis, and dry eye disease (Lee et al., 2016; Hagan et al., 2018).

Another study (Wang et al., 2008) reported that FGR (ENSP00000363115) may also participate in eye diseases, including age-related macular degeneration and uveitis.

\section{Genes With G-Protein Coupled Receptor Binding (GO:0001664) Capacity}

Apart from being proliferation regulatory genes in immune cells, JAK1 and JAK3, the next inferred gene, are also members of a functional WNT gene family WNT16 (ENSP00000222462). For encoding of a secreted signaling protein, such gene encodes a ligand for the members of frizzled family of seven transmembrane receptors (Wergedal et al., 2015; Ozeki et al., 2016). For its specific contribution on the pathogenic approach of uveitis, no direct evidence confirmed that WNT16 participates in uveitis-specific pathogenesis. However, two recent studies (Reischl et al., 2007; Nalesso et al., 2017) confirmed that WNT16, together with MNT5, participate in the pathogenesis of uveitis by interfering immune responses.

A homolog of WNT16, WNT3 (ENSP00000225512) was also predicted to participate in uveitis-specific pathogenesis. According to a recent publication on the genetic components of wnt/ $\beta$-catenin signaling pathway (Nakatsu et al., 2011), this gene is similar to our predicted biomarker WNT3 and regulates the proliferation of eye cells, including urea cells (Nakatsu et al., 2011). The abnormal proliferation of urea cells, especially immune cells, may trigger the initiation and proliferation of uveitis. Therefore, speculating that WNT16 and WNT3 may be functionally related to uveitis is reasonable.

The next inferred gene named WNT7B (ENSP00000341032) is also a specific member of $w n t / \beta$-catenin signaling pathway. According to the same literature mentioned above, such gene participating in $w n t / \beta$-catenin signaling pathway may also contribute to the pathogenic progressions of uveitis due to its abnormal regulatory role on urea immune cell proliferation in situ. As a homolog of WNT7B, WNT7A (ENSP00000285018) acts as one of the potential pathogenic factors of uveitis by interfering immune cell proliferation and maturation. Similarly, WNT2B (ENSP00000358698), WNT9A (ENSP00000272164), WNT4 (ENSP00000290167), and WNT2 (ENSP00000265441) all participate in the pathogenesis of uveitis through the regulation of urea cells, thus validating their strong relationships with uveitis.

We screened out eight WNT signaling pathway components that may contribute to the pathogenesis of uveitis. According to recent publications, our inferred genes (WNT2, WNT16, 
WNT3, WNT7A, and WNT7B) are functionally related to the initiation and progression of uveitis due to their interference to the proliferation of urea and mixed immune cells.

\section{Other Functional Inferred Uveitis-Related Genes} GLI2 (ENSP00000354586) was also regarded as a potential pathogenic gene of uveitis. Such gene has been widely reported to act as a transcriptional regulator by encoding a $\mathrm{C} 2 \mathrm{H} 2$-type zinc finger protein (Eichberger et al., 2006; Jackson et al., 2015). For its specific contribution on uveitis, GLI2 regulates NotchGli2 axis and hedgehog signaling pathway; however, no direct reports confirmed its pathogenic role (Roessler et al., 2003; Ringuette et al., 2016). In the pathogenic conditions of urea tissues, the inflammatory environment is also regulated by such two pathways (Takezaki et al., 2011; Swiderska-Syn et al., 2013). Therefore, this gene can be regarded as a potential uveitis-related gene.

ZAP70 (ENSP00000264972) is also a functional inferred uveitis-related gene. According to a recent publication, ZAP70 is a potential biomarker instructing the onset of uveitis in mouse models (Kleinwort et al., 2016). Therefore, this gene can be inferred as a uveitis-related gene.

MAPK8 (ENSP00000353483) was inferred to be potential uveitis-related gene, participating in the specific pathogenesis of such disease. It is necessary to point out that this gene was also reported in one previous study ( $\mathrm{Lu}$ et al., 2017). Early in 2014, a systematic study (Lisanti et al., 2014) on tumor stroma confirmed that MAPK8 contribute to the pathogenesis of a typical tumor complication, uveitis, corresponding with our prediction. In the same year, another independent study (Honke et al., 2014) validated that p38-MAPK8 participated in the specific IL-6 mediated inflammatory responses. Therefore, such

\section{REFERENCES}

Akyol, L., Aslan, K., Ozgen, M., and Sayarlioglu, M. (2015). Bilateral sacroiliitis and uveitis comorbidity: brucellosis? Ankylosing spondylitis? BMJ Case Rep. 2015:bcr2015211461. doi: 10.1136/bcr-2015-211461

Barabasi, A. L., Gulbahce, N., and Loscalzo, J. (2011). Network medicine: a network-based approach to human disease. Nat. Rev. Genet. 12, 56-68. doi: $10.1038 / \mathrm{nrg} 2918$

Bogaert, D., Van Schil, K., Taghon, T., Bordon, V., Bonroy, C., Dullaers, M., et al. (2016). Persistent rotavirus diarrhea post-transplant in a novel JAK3-SCID patient after vaccination. Pediatr. Allergy Immunol. 27, 93-96. doi: /10.1111/ pai. 12455

Bojanova, M., Bodaghi, B., Hannachi, N., Jouffroy, T., Fel, A., Le Hoang, P., et al. (2013). Measure of herpesvirus-specific ocular antibody production in patients with uveitis. J. Clin. Virol. 58, 718-721. doi: 10.1016/j.jcv.2013.10.013

Cai, Y.-D., Zhang, Q., Zhang, Y.-H., Chen, L., and Huang, T. (2017). Identification of genes associated with breast cancer metastasis to bone on a protein-protein interaction network with a shortest path algorithm. J. Proteome Res. 16, 1027-1038. doi: 10.1021/acs.jproteome.6b00950

Chen, L., Liu, T., and Zhao, X. (2018a). Inferring anatomical therapeutic chemical (ATC) class of drugs using shortest path and random walk with restart algorithms. Biochim. Biophys. Acta 1864, 2228-2240. doi: 10.1016/j.bbadis.2017. 12.019

Chen, L., Pan, X., Hu, X., Zhang, Y.-H., Wang, S., Huang, T., et al. (2018b). Gene expression differences among different MSI statuses in colorectal cancer. Int. J. Cancer [Epub ahead of print].

Chen, L., Zhang, Y.-H., Zhang, Z., Huang, T., and Cai, Y.-D. (2018c). Inferring novel tumor suppressor genes with a protein-protein interaction network identified gene MAPK8 may also be a specific uveitis associated gene, corresponding with previous studies and publications.

Literature-based analysis confirmed some of the inferred genes as participating in uveitis, thus validating that our results are reliable. The rest of the inferred genes were left for readers. Most of them are suggested to be related to uveitis.

\section{CONCLUSION}

This study aims to infer novel uveitis-related genes. An efficient network algorithm, LHD algorithm, was adopted as the basic searching algorithm and was executed on a PPI network using validated uveitis-related genes as seed nodes. With the help of three screening tests, 59 functional genes were finally accessed. These novel inferred genes can be useful materials to uncover the pathogenesis of uveitis.

\section{AUTHOR CONTRIBUTIONS}

All authors contributed to the research and reviewed the manuscript. MY designed the study. SL, KZ, XW, and HL performed the experiments. SL, KZ, XA, and YX analyzed the results. SL and KZ wrote the manuscript.

\section{SUPPLEMENTARY MATERIALS}

The Supplementary Material for this article can be found online at: https://www.frontiersin.org/articles/10.3389/fgene. 2018.00425/full\#supplementary-material

and network diffusion algorithms. Mol. Ther. Methods Clin. Dev. 10, 57-67. doi: 10.1016/j.omtm.2018.06.007

Chen, L., Xing, Z., Huang, T., Shu, Y., Huang, G., and Li, H.-P. (2016a). Application of the shortest path algorithm for the discovery of breast cancer related genes. Curr. Bioinformatics 11, 51-58. doi: 10.2174/157489361166615111922 0024

Chen, L., Zhang, Y.-H., Zheng, M., Huang, T., and Cai, Y.-D. (2016b). Identification of compound-protein interactions through the analysis of gene ontology, KEGG enrichment for proteins and molecular fragments of compounds. Mol. Genet. Genomics 291, 2065-2079.

Chen, L., Yang, J., Xing, Z., Yuan, F., Shu, Y., Zhang, Y., et al. (2017a). An integrated method for the identification of novel genes related to oral cancer. PLoS One 12:e0175185. doi: 10.1371/journal.pone.0175185

Chen, L., Zhang, Y.-H., Lu, G., Huang, T., and Cai, Y.-D. (2017b). Analysis of cancer-related lncRNAs using gene ontology and KEGG pathways. Artif. Intell. Med. 76, 27-36. doi: 10.1016/j.artmed.2017.02.001

Corneth, O. B. J., Klein Wolterink, R. G. J., and Hendriks, R. W. (2016). BTK signaling in B cell differentiation and autoimmunity. Curr. Top. Microbiol. Immunol. 393, 67-105. doi: 10.1007/82_2015_478

Eichberger, T., Sander, V., Schnidar, H., Regl, G., Kasper, M., Schmid, C., et al. (2006). Overlapping and distinct transcriptional regulator properties of the GLI1 and GLI2 oncogenes. Genomics 87, 616-632. doi: 10.1016/j.ygeno.2005. 12.003

Gao, P., Wang, Q. P., Chen, L., and Huang, T. (2012). Prediction of human genes regulatory functions based on proteinprotein interaction network. Protein Pept. Lett. 19, 910-916. doi: 10.2174/092986612802084528

Gao, Y. F., Chen, L., Cai, Y. D., Feng, K. Y., Huang, T., and Jiang, Y. (2012). Predicting metabolic pathways of small molecules and enzymes based 
on interaction information of chemicals and proteins. PLoS One 7:e45944. doi: 10.1371/journal.pone.0045944

Gormen, T. H., Leiserson, C. E., Rivest, R. L., and Stein, C. (eds). (1990). Introduction to Algorithms. Cambridge, MA: MIT press.

Gui, T., Dong, X., Li, R., Li, Y., and Wang, Z. (2015). Identification of hepatocellular carcinoma-related genes with a machine learning and network analysis. J. Comput. Biol. 22, 63-71. doi: 10.1089/cmb.2014.0122

Hagan, S., Fyfe, M. C. T., Ofori-Frimpong, B., Oliver, K., Foster, M. R., Sirohi, S., et al. (2018). Narrow spectrum kinase inhibitors demonstrate promise for the treatment of dry eye disease and other ocular inflammatory disorders. Invest. Ophthalmol. Vis. Sci. 59, 1443-1453. doi: 10.1167/iovs.17-23479

Hauck, F., Blumenthal, B., Fuchs, S., Lenoir, C., Martin, E., Speckmann, C., et al. (2015). SYK expression endows human ZAP70-deficient CD8 T cells with residual TCR signaling. Clin. Immunol. 161, 103-109. doi: 10.1016/j.clim.2015. 07.002

Honke, N., Ohl, K., Wiener, A., Bierwagen, J., Peitz, J., Di Fiore, S., et al. (2014). The p38-mediated rapid down-regulation of cell surface gp130 expression impairs interleukin-6 signaling in the synovial fluid of juvenile idiopathic arthritis patients. Arthritis Rheumatol. 66, 470-478. doi: 10.1002/art.38245

Hu, L. L., Huang, T., Liu, X. J., and Cai, Y. D. (2011a). Predicting protein phenotypes based on protein-protein interaction network. PLoS One 6:e17668. doi: 10.1371/journal.pone.0017668

Hu, L. L., Huang, T., Shi, X., Lu, W. C., Cai, Y. D., and Chou, K. C. (2011b). Predicting functions of proteins in mouse based on weighted proteinprotein interaction network and protein hybrid properties. PLoS One 6:e14556. doi: 10.1371/journal.pone.0014556

Huang, G., Chu, C., Huang, T., Kong, X., Zhang, Y., Zhang, N., et al. (2016). Exploring mouse protein function via multiple approaches. PLoS One 11:e0166580. doi: 10.1371/journal.pone.0166580

Ildefonso, C. J., Jaime, H., Biswal, M. R., Boye, S. E., Li, Q., Hauswirth, W. W., et al. (2015). Gene therapy with the caspase activation and recruitment domain reduces the ocular inflammatory response. Mol. Ther. 23, 875-884. doi: 10.1038/ mt.2015.30

Jackson, D. A., Smith, T. D., Amarsaikhan, N., Han, W., Neil, M. S., Boi, S. K., et al. (2015). Modulation of the IL-6 receptor alpha underlies GLI2-mediated regulation of ig secretion in waldenstrom macroglobulinemia cells. J. Immunol. 195, 2908-2916. doi: 10.4049/jimmunol.1402974

Junqueira, D. L., Lopes, F. S., Biteli, L. G., and Prata, T. S. (2013). Pattern of inner retinal layers involvement in pigmented paravenous retinochoroidal atrophy as determined by SD-OCT: case report. Arq. Bras. Oftalmol. 76, 380-382. doi: 10.1590/S0004-27492013000600014

Kalinina Ayuso, V., Makhotkina, N., Van Tent-Hoeve, M., De Groot-Mijnes, J. D., Wulffraat, N. M., Rothova, A., et al. (2014). Pathogenesis of juvenile idiopathic arthritis associated uveitis: the known and unknown. Surv. Ophthalmol. 59, 517-531. doi: 10.1016/j.survophthal.2014.03.002

Kleinwort, K. J., Amann, B., Hauck, S. M., Feederle, R., Sekundo, W., and Deeg, C. A. (2016). Immunological characterization of intraocular lymphoid follicles in a spontaneous recurrent uveitis model. Invest. Ophthalmol. Vis. Sci. 57, 4504-4511. doi: 10.1167/iovs.16-19787

Kohler, S., Bauer, S., Horn, D., and Robinson, P. N. (2008). Walking the interactome for prioritization of candidate disease genes. Am. J. Hum. Genet. 82, 949-958. doi: 10.1016/j.ajhg.2008.02.013

Lee, E. J., Brown, B. R., Vance, E. E., Snow, P. E., Silver, P. B., Heinrichs, D., et al. (2016). Mincle activation and the Syk/Card9 signaling axis are central to the development of autoimmune disease of the eye. J. Immunol. 196, 3148-3158. doi: 10.4049/jimmunol.1502355

Lee, J. E., Lee, A. S., Kim, D. H., Jung, Y. J., Lee, S., Park, B. H., et al. (2012). Janex-1, a JAK3 inhibitor, ameliorates tumor necrosis factor-alpha-induced expression of cell adhesion molecules and improves myocardial vascular permeability in endotoxemic mice. Int. J. Mol. Med. 29, 864-870. doi: 10.3892/ijmm.2012.920

Leiserson, M. D., Vandin, F., Wu, H. T., Dobson, J. R., Eldridge, J. V., Thomas, J. L., et al. (2015). Pan-cancer network analysis identifies combinations of rare somatic mutations across pathways and protein complexes. Nat. Genet. 47, 106-114. doi: 10.1038/ng.3168

Li, J., Chen, L., Wang, S., Zhang, Y., Kong, X., Huang, T., et al. (2018a). A computational method using the random walk with restart algorithm for identifying novel epigenetic factors. Mol. Genet. Genomics 293, 293-301. doi: 10.1007/s00438-017-1374-5
Li, J., Lu, L., Zhang, Y., Liu, M., Chen, L., Huang, T., et al. (2018b). Identification of synthetic lethality based on a functional network by using machine learning algorithms. J. Cell. Biochem. [Epub ahead of print].

Li, L., Wang, Y., An, L., Kong, X., and Huang, T. (2017). A network-based method using a random walk with restart algorithm and screening tests to identify novel genes associated with Meniere's disease. PLoS One 12:e0182592. doi: 10.1371/ journal.pone. 0182592

Li, Y., and Li, J. (2012). Disease gene identification by random walk on multigraphs merging heterogeneous genomic and phenotype data. BMC Genomics 13:S27. doi: 10.1186/1471-2164-13-S7-S27

Liao, H. T., Li, T. H., Chen, C. H., Chen, H. A., Chen, W. S., Lai, C. C., et al. (2018) Janus kinase- 1 and 3 in ankylosing spondylitis. J. Formos. Med. Assoc. [Epub ahead of print].

Lim, F. P., Wong, C. W., Loh, B. K., Chan, C. M., Yeo, I., Lee, S. Y., et al. (2016). Prevalence and clinical correlates of focal choroidal excavation in eyes with age-related macular degeneration, polypoidal choroidal vasculopathy and central serous chorioretinopathy. Br. J. Ophthalmol. 100, 918-923. doi: 10.1136/ bjophthalmol-2015-307055

Lisanti, M. P., Tsirigos, A., Pavlides, S., Reeves, K. J., Peiris-Pages, M., Chadwick, A. L., et al. (2014). JNK1 stress signaling is hyper-activated in high breast density and the tumor stroma: connecting fibrosis, inflammation, and stemness for cancer prevention. Cell Cycle 13, 580-599. doi: 10.4161/cc.27379

Liu, L., Chen, L., Zhang, Y.-H., Wei, L., Cheng, S., Kong, X., et al. (2016). Analysis and prediction of drug-drug interaction by minimum redundancy maximum relevance and incremental feature selection. J. Biomol. Struct. Dyn. 35, 312-329. doi: 10.1080/07391102.2016.1138142

Loureiro, A. P., Hamond, C., and Lilenbaum, W. (2013). Leptospirosis in horses. Vet. Rec. 172, 479-480. doi: 10.1136/vr.f2824

Lu, S., Yan, Y., Li, Z., Chen, L., Yang, J., Zhang, Y., et al. (2017). Determination of genes related to uveitis by utilization of the random walk with restart algorithm on a protein-protein interaction network. Int. J. Mol. Sci. 18:E1045. doi: 10.3390/ijms 18051045

Luger, D., Yang, Y. A., Raviv, A., Weinberg, D., Banerjee, S., Lee, M. J., et al. (2013). Expression of the B-cell receptor component CD79a on immature myeloid cells contributes to their tumor promoting effects. PLoS One 8:e76115. doi: 10.1371/journal.pone.0076115

Nagel, D., Bognar, M., Eitelhuber, A. C., Kutzner, K., Vincendeau, M., and Krappmann, D. (2015). Combinatorial BTK and MALT1 inhibition augments killing of CD79 mutant diffuse large B cell lymphoma. Oncotarget 6, 4223242242. doi: 10.18632/oncotarget.6273

Nakatsu, M. N., Ding, Z., Ng, M. Y., Truong, T. T., Yu, F., and Deng, S. X. (2011). Wnt/beta-catenin signaling regulates proliferation of human cornea epithelial stem/progenitor cells. Invest. Ophthalmol. Vis. Sci. 52, 4734-4741. doi: $10.1167 /$ iovs.10-6486

Nalesso, G., Thomas, B. L., Sherwood, J. C., Yu, J., Addimanda, O., Eldridge, S. E., et al. (2017). WNT16 antagonises excessive canonical WNT activation and protects cartilage in osteoarthritis. Ann. Rheum. Dis. 76, 218-226. doi: 10.1136/annrheumdis-2015-208577

Notarangelo, L. D., Mella, P., Jones, A., De Saint Basile, G., Savoldi, G., Cranston, T., et al. (2001). Mutations in severe combined immune deficiency (SCID) due to JAK3 deficiency. Hum. Mutat. 18, 255-263. doi: 10.1002/humu. 1188

Ozeki, N., Mogi, M., Hase, N., Hiyama, T., Yamaguchi, H., Kawai, R., et al. (2016). Wnt16 signaling is required for IL-1beta-induced matrix metalloproteinase-13regulated proliferation of human stem cell-derived osteoblastic cells. Int. J. Mol. Sci. 17:221. doi: 10.3390/ijms17020221

Pan, J., Kapur, M., and Mccallum, R. (2014). Noninfectious immune-mediated uveitis and ocular inflammation. Curr. Allergy Asthma Rep. 14:409. doi: 10.1007/s11882-013-0409-1

Pan, X., Hu, X., Zhang, Y.-H., Feng, K., Wang, S. P., Chen, L., et al. (2018). Identifying patients with atrioventricular septal defect in down syndrome populations by using self-normalizing neural networks and feature selection. Genes 9:208. doi: 10.3390/genes9040208

Reischl, J., Schwenke, S., Beekman, J. M., Mrowietz, U., Sturzebecher, S., and Heubach, J. F. (2007). Increased expression of Wnt5a in psoriatic plaques. J. Invest. Dermatol. 127, 163-169. doi: 10.1038/sj.jid.5700488

Rekas, M., Barchan-Kucia, K., Konopinska, J., Mariak, Z., and Zarnowski, T. (2015). Analysis and modeling of anatomical changes of the anterior segment 
of the eye after cataract surgery with consideration of different phenotypes of eye structure. Curr. Eye Res. 40, 1018-1027. doi: 10.3109/02713683.2014.97 5366

Ringuette, R., Atkins, M., Lagali, P. S., Bassett, E. A., Campbell, C., Mazerolle, C., et al. (2016). A notch-Gli2 axis sustains Hedgehog responsiveness of neural progenitors and Muller glia. Dev. Biol. 411, 85-100. doi: 10.1016/j.ydbio.2016. 01.006

Roessler, E., Du, Y. Z., Mullor, J. L., Casas, E., Allen, W. P., Gillessen-Kaesbach, G., et al. (2003). Loss-of-function mutations in the human GLI2 gene are associated with pituitary anomalies and holoprosencephaly-like features. Proc. Natl. Acad. Sci. U.S.A. 100, 13424-13429. doi: 10.1073/pnas.2235734100

Rosenbaum, J. T. (2015). Uveitis in spondyloarthritis including psoriatic arthritis, ankylosing spondylitis, and inflammatory bowel disease. Clin. Rheumatol. 34, 999-1002. doi: 10.1007/s10067-015-2960-8

Sen, E. S., Dick, A. D., and Ramanan, A. V. (2015). Uveitis associated with juvenile idiopathic arthritis. Nat. Rev. Rheumatol. 11, 338-348. doi: 10.1038/nrrheum. 2015.20

Stark, C., Breitkreutz, B.-J., Reguly, T., Boucher, L., Breitkreutz, A., and Tyers, M. (2006). BioGRID: a general repository for interaction datasets. Nucleic Acids Res. 34, D535-D539. doi: 10.1093/nar/gkj109

Swiderska-Syn, M., Suzuki, A., Guy, C. D., Schwimmer, J. B., Abdelmalek, M. F., Lavine, J. E., et al. (2013). Hedgehog pathway and pediatric nonalcoholic fatty liver disease. Hepatology 57, 1814-1825. doi: 10.1002/hep. 26230

Takezaki, T., Hide, T., Takanaga, H., Nakamura, H., Kuratsu, J., and Kondo, T. (2011). Essential role of the Hedgehog signaling pathway in human gliomainitiating cells. Cancer Sci. 102, 1306-1312. doi: 10.1111/j.1349-7006.2011. 01943.x

Tang, W., Wan, S., Yang, Z., Teschendorff, A. E., and Zou, Q. (2017). Tumor origin detection with tissue-specific miRNA and DNA methylation markers. Bioinformatics 34, 398-406.

Urban, B., Bakunowicz-Lazarczyk, A., and Michalczuk, M. (2014). Immune recovery uveitis: pathogenesis, clinical symptoms, and treatment. Mediators Inflamm. 2014:971417. doi: 10.1155/2014/971417

Vargas, L., Hamasy, A., Nore, B. F., and Smith, C. I. (2013). Inhibitors of BTK and ITK: state of the new drugs for cancer, autoimmunity and inflammatory diseases. Scand. J. Immunol. 78, 130-139. doi: 10.1111/sji.12069

Wang, D., Li, J.-R., Zhang, Y.-H., Chen, L., Huang, T., and Cai, Y.-D. (2018). Identification of differentially expressed genes between original breast cancer and xenograft using machine learning algorithms. Genes 9:E155. doi: 10.3390/ genes 9030155

Wang, J., Ohno-Matsui, K., Yoshida, T., Kojima, A., Shimada, N., Nakahama, K., et al. (2008). Altered function of factor I caused by amyloid beta: implication for pathogenesis of age-related macular degeneration from Drusen. J. Immunol. 181, 712-720. doi: 10.4049/jimmunol.181.1.712

Wang, X., Khanna, N., Wu, J., Godri Pollitt, K., Evans, G. J., Chow, C. W., et al. (2015). Syk mediates airway contractility independent of leukocyte function. Allergy 70, 429-435. doi: 10.1111/all.12564
Wergedal, J. E., Kesavan, C., Brommage, R., Das, S., and Mohan, S. (2015). Role of WNT16 in the regulation of periosteal bone formation in female mice. Endocrinology 156, 1023-1032. doi: 10.1210/en.2014-1702

Wu, H., Wang, W., Liu, F., Weisberg, E. L., Tian, B., Chen, Y., et al. (2014). Discovery of a potent, covalent BTK inhibitor for B-cell lymphoma. ACS Chem. Biol. 9, 1086-1091. doi: 10.1021/cb4008524

Xenarios, I., Rice, D. W., Salwinski, L., Baron, M. K., Marcotte, E. M., and Eisenberg, D. (2000). DIP: the database of interacting proteins. Nucleic Acids Res. 28, 289-291. doi: 10.1093/nar/28.1.289

Xu, D., Hou, S., Jiang, Y., Zhang, J., Cao, S., Zhang, D., et al. (2015). Complement C5 gene confers risk for acute anterior uveitis. Invest. Ophthalmol. Vis. Sci. 56, 4954-4960. doi: 10.1167/iovs.15-16645

Yang, M. M., Lai, T. Y., Tam, P. O., Chiang, S. W., Chan, C. K., Luk, F. O., et al. (2012). Complement factor $\mathrm{H}$ and interleukin gene polymorphisms in patients with non-infectious intermediate and posterior uveitis. Mol. Vis. 18, 1865-1872.

Yu, H., Liu, Y., Zhang, L., Wu, L., Zheng, M., Cheng, L., et al. (2014). FoxO1 gene confers genetic predisposition to acute anterior uveitis with ankylosing spondylitis. Invest. Ophthalmol. Vis. Sci. 55, 7970-7974. doi: 10.1167/iovs.1415460

Yuan, F., and Lu, W. (2017). Prediction of potential drivers connecting different dysfunctional levels in lung adenocarcinoma via a protein-protein interaction network. Biochim. Biophys. Acta 1864, 2284-2293. doi: 10.1016/j.bbadis.2017. 11.018

Zeng, X., Liao, Y., Liu, Y., and Zou, Q. (2017). Prediction and validation of disease genes using HeteSim Scores. IEEE/ACM Trans. Comput. Biol. Bioinform. 14, 687-695. doi: 10.1109/TCBB.2016.2520947

Zeng, X., Liu, L., Lu, L., and Zou, Q. (2018). Prediction of potential diseaseassociated microRNAs using structural perturbation method. Bioinformatics 34 , 2425-2432. doi: 10.1093/bioinformatics/bty112

Zhang, J., Suo, Y., Liu, M., and Xu, X. (2017). Identification of genes related to proliferative diabetic retinopathy through RWR algorithm based on proteinprotein interaction network. Biochim. Biophys. Acta 1864, 2369-2375. doi: 10. 1016/j.bbadis.2017.11.017

Zhang, J., Yang, J., Huang, T., Shu, Y., and Chen, L. (2016). Identification of novel proliferative diabetic retinopathy related genes on protein-protein interaction network. Neurocomputing 217, 63-72. doi: 10.1016/j.neucom.2015.09.136

Conflict of Interest Statement: The authors declare that the research was conducted in the absence of any commercial or financial relationships that could be construed as a potential conflict of interest.

Copyright (C) $2018 \mathrm{Lu}$, Zhao, Wang, Liu, Ainiwaer, Xu and Ye. This is an open-access article distributed under the terms of the Creative Commons Attribution License (CC BY). The use, distribution or reproduction in other forums is permitted, provided the original author(s) and the copyright owner(s) are credited and that the original publication in this journal is cited, in accordance with accepted academic practice. No use, distribution or reproduction is permitted which does not comply with these terms. 